\title{
Operation Planning of the Elective Patients in an Orthopedic Surgery Department
}

\author{
Daniel Clavel $^{1}$, Cristian Mahulea ${ }^{1}$, Jorge Albareda ${ }^{2}$ Manuel Silva ${ }^{1}$ \\ ${ }^{1}$ Afiliación: Grupo de Ingeniería de Sistemas de Eventos Discretos (GISED) \\ Instituto de Investigación en Ingeniería de Aragón (I3A) \\ Universidad de Zaragoza, Mariano Esquillor s/n, 50018, Zaragoza, Spain. \\ Tel.+34-976762707, e-mail: clavel@unizar.es \\ ${ }^{2}$ Afiliación: Servicio de Cirugía Ortopédica y Traumatológica, Hospital Clínico “Lozano Blesa”, Zaragoza (España)
}

\begin{abstract}
This paper considers the operation scheduling and planning of elective patients in the Orthopedic Department of the "Lozano Blesa" Hospital in Zaragoza. We assume an ordered list of patients that should be planned for surgery. We propose a mixed linear integer programming problem to obtain a utilization rate of $80 \%$ per room. The results are tested on some real data from the hospital and some simulation results are provided.
\end{abstract}

\section{Introduction}

In this paper, we use mathematical programming to model the planning of non-urgent surgeries in a hospital department. This can be seen as the planing of a production system: (a) first, there is a waiting list of patients who represent the system demand and (b) second, there exists a limited numbers of surgeons and a limited number of operations rooms (OR) that represents the capacity of the production system. For our application, the bottleneck of the resources are the OR. In particular, there exists two OR for nonemergency surgeries in the Orthopedic Department, each one being available only 7 hours per day.

The objective of the proposed approach is to obtain an occupation rate of 80 percent. In order to solve this problem, we obtain a mathematical model based on linear programing that allows doctors to plan the surgeries with a determined occupation rate of $80 \%$ respecting, if possible, the order of patients in the waiting list.

This model will be used to propose a Decision Support System (DSS) to be used in the management of the Orthopedic Department of the "Lozano Blesa" Hospital in Zaragoza. The DSS can be used to: (a) optimize the use of OR and computerizing the surgeries allocation method, (b) estimate the necessary ORs to perform all surgeries in the waiting list and (c) dynamic updated the input parameters of the model to improve the solution.

\section{Organizational structure of the Orthopedic Department}

This department is composed by five medical teams. Each team consists of seven doctors and has a coordinator (the more experienced doctor), each doctor having his own waiting list of patients waiting for surgery. The patients belonging to the waiting lists of the doctors belonging to the same team composed the patient waiting list of the team.

The Orthopedic Department have two OR available per day for non-urgent surgeries. Each of these OR have an active schedule from 8 am to $3 \mathrm{pm}$ ( 7 hours). The Orthopedic Department is organized in such way that during a same day each OR is used by the same team. The head of the Orthopedic Department (that is a medical doctor as well) is responsible for assigning teams to the OR. Each team has a coordinator who is responsible of operation planning of his team.

It is important that the real occupation of the OR does not exceed $100 \%$ of the available time because in this case medical staff could lengthen their working day. With the propose of preventing these situations, the health system considers appropriated an occupancy rate of 80 percent. This occupancy rate allows, on one hand, the sterilization of operating rooms after each surgery and, on the other hand, absorb possible surgeries delays.

In this way, the problem that we solve is the following: Problem 1: Given a list of patients that should be planned for surgery, schedule the next $m$ working days of ORs with an occupancy rate near of 80 percent, respecting as far as possible the order of the patients in the waiting list. 
Using the history datas from the last two years, for each pathology we compute the average duration for surgery.

Let us define the input numerical data of the problem $V_{e}$ as a matrix of dimension [2xn] where $\mathrm{n}$ is the size of the list. The first row of $V_{e}$ is the preference order (denoted also as $V_{e 1}$ ) and the second row is the average duration associated whit the corresponding pathology $\left(V_{e 2}\right)$.

\section{MILP formulation}

To solve the problem described in the previous section, we proposed an Mixed Interger Linear Programing (MILP) problem. Let us define the following variables,

- $\boldsymbol{S}_{1}, \boldsymbol{S}_{2}, \boldsymbol{S}_{3}, \ldots \boldsymbol{S}_{m}$ vectors of binary variables where $m$ is the number of working days to planning. Each $\boldsymbol{S}_{i} \in\{0,1\}^{n}$ is a vector of $n$ binary variables where $n$ is the size of the waiting list. $\boldsymbol{S}_{i}[j]=1$ means that surgery $j$ is operated in the $i^{\text {th }}$ working day;

- $\quad \alpha \in \mathfrak{R}_{\geq 0}^{m}$ is a vector of absolute deviations (in minutes)of each day with respect to the objective occupation.

Let us define the following MILP:

Min

$\sum_{i=1}^{m}\left[\alpha_{i} *(m-i-1)+\beta * \boldsymbol{V}_{e 1} * \boldsymbol{S}_{i} *(m-i-1)\right]$

Subject to:

$$
\left\{\begin{aligned}
\boldsymbol{V}_{e 2} * \boldsymbol{S}_{i}-336 & \leq \alpha_{i}, \quad \forall \mathrm{i}=1,2, \ldots, \mathrm{m} \\
-\boldsymbol{V}_{e 2} * \boldsymbol{S}_{i}+336 & \leq \alpha_{i}, \\
\sum_{i=1}^{m} \boldsymbol{S}_{i}[j] & \leq 1, \quad \forall \mathrm{j}=1,2, \ldots, \mathrm{n}
\end{aligned}\right.
$$

The cost function is composed by two terms: the first one is related to the objective occupancy rate while the second with the preference order of the list.

\section{Decision Support System for Operation Planning}

In order to perform a rapid, efficient and dynamic operation planning we propose a decision support system (DSS) to help the managers in the operation planning. The core of the DSS is the MILP presented in previous section to operation planning, but also it includes other features that enable a) updating the waiting list, b) dynamic planning and, c) improving the input data by updating the historical data.

The flowchart of the DSS is given in Fig. 1 and starts by adding a new patient to waiting lists. When a team coordinator decides to plan the next $\mathrm{m}$ working days, the tool assigns an average theoretical duration to each surgery in the waiting list in function of the pathology and the doctor. In this way, the vector $\mathrm{Ve}$ is generated. Then, the operation planning is performed in an iterative way (as is described in IV-B). The states in the list of the surgeries that have been planned change from pending to plan. Once a specific surgery is performed, the surgeon introduces the time spent on the surgery in the tool. This new input data is used to update the average duration (as is described in IV-C). Additionally, the tool removes the patients that have been operated from the waiting list. If a planed surgery finally is not performed, DSS changes the state of this surgery from planned to pending.

\section{Simulation results using CPLEX}

In this section we are going to test the behavior of the MILP. In particular we validate the model and we show the influence of the parameter $\beta$ on the resulted planning.

Assuming a list of 300 patients and different values of $\beta$ we have planned 60 working days. The results obtained of occupation rate have been analyzed. Table I shows a statistic analysis of planned occupation rate including the average, standard deviation and the extreme values. Table II shows the planning done for the first 5 working days of OR with different values of $\beta$.

\section{Conclusions}

By modeling and solving MILP it is possible to perform surgical operations planning of elective patients with the objective of maximizing the occupation rate of the OR.

The simulations performed conclude that the computational time of solving MILP is affected mainly by the number of planning days. To schedule 
more than 8 days in a reasonable time is necessary to solve the problem in an iterative way. Design parameter $\beta$ establishes a compromise between the occupation rate and the order of patients in the waiting list.

This paper presents a DSS, which by using MILP, helps the managers in the operation planning and scheduling. It is includes features that enables dynamic planning and enables the improvement of the input data. The DSS has been tested by using real data from the hospital and as future work we plan to integrate the DSS in the hospital department.

\section{TABLES}

Tabla I. Statistic analysis of occupation rate

\begin{tabular}{|c|c|c|c|c|}
\hline$\beta$ & Average & Deviation & Minimum & Maximum \\
\hline \hline 3 & 79.278 & 1.554 & 70.952 & 81.667 \\
\hline 2 & 80.340 & 1.033 & 77.619 & 81.905 \\
\hline 1.5 & 80.340 & 0.932 & 77.857 & 81.905 \\
\hline 1 & 80.340 & 0.719 & 78.333 & 81.667 \\
\hline$\frac{2}{3}$ & 80.183 & 0.703 & 78.333 & 81.667 \\
\hline 0.5 & 80.238 & 0.624 & 78.333 & 81.190 \\
\hline$\frac{1}{3}$ & 80.238 & 0.621 & 78.571 & 81.905 \\
\hline
\end{tabular}

Tabla II. 5 First ORs working days of the planning of a same list with different values of parameter $\beta$

\begin{tabular}{|c|c|c|c|c|c|}
\hline$\beta$ & Day & Opl & Op2 & Op3 & Occupation \\
\hline \multirow{5}{*}{3} & $\mathrm{~T}$ & $T$ & $\frac{1}{2}$ & $\frac{1}{4}$ & 77.857 \\
\hline & 2 & 3 & 5 & 6 & 80.714 \\
\hline & 3 & 7 & 8 & 10 & 80.714 \\
\hline & 4 & 9 & 17 & 0 & 70.952 \\
\hline & 5 & $\mathrm{~T} 2$ & 13 & 14 & 80.714 \\
\hline \multirow{5}{*}{2} & $T$ & $T$ & 2 & $\overline{6}$ & 80.714 \\
\hline & 2 & 3 & 5 & 9 & 81.666 \\
\hline & 3 & 7 & 8 & T0 & 80.714 \\
\hline & 4 & 4 & 13 & 18 & 80.238 \\
\hline & 5 & $\pi$ & 12 & 15 & 79.524 \\
\hline \multirow{5}{*}{1.5} & $\overline{1}$ & $\overline{11}$ & $\overline{2}$ & 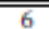 & 80.714 \\
\hline & 2 & 3 & 5 & 9 & 81.666 \\
\hline & 3 & 7 & 8 & TO & 80.714 \\
\hline & 4 & 4 & 13 & 18 & 80.238 \\
\hline & 5 & $\pi$ & $T 2$ & 15 & 79.524 \\
\hline \multirow{5}{*}{1} & $\overline{1}$ & 1 & 2 & $\overline{6}$ & 80.714 \\
\hline & 2 & 3 & $T$ & 10 & 80.714 \\
\hline & 3 & 5 & 8 & y & 81.666 \\
\hline & 4 & 4 & 13 & 18 & 80.238 \\
\hline & 5 & TI & 12 & 15 & 79.524 \\
\hline \multirow{5}{*}{$\frac{2}{3}$} & $T$ & $T$ & 2 & $\overline{6}$ & 80.714 \\
\hline & 2 & 3 & 7 & 10 & 80.714 \\
\hline & 3 & 5 & 8 & 9 & 81.666 \\
\hline & 4 & 4 & 13 & 18 & 80.238 \\
\hline & 5 & $\pi$ & 12 & 15 & 79.524 \\
\hline \multirow{5}{*}{0.5} & $T$ & $T$ & 2 & $\overline{6}$ & 80.714 \\
\hline & 2 & 3 & $T$ & 10 & 80.714 \\
\hline & 3 & 5 & Tा & 15 & 79.524 \\
\hline & 4 & 4 & 13 & 18 & 80.238 \\
\hline & 5 & 8 & 14 & 16 & 80.714 \\
\hline \multirow{5}{*}{$\frac{1}{3}$} & $\mathrm{~T}$ & $T$ & 2 & 6 & 80.714 \\
\hline & 2 & 3 & 7 & T0 & 80.714 \\
\hline & 3 & 5 & 11 & 15 & 79.524 \\
\hline & 4 & 4 & 13 & 18 & 80.238 \\
\hline & 5 & 8 & 9 & 29 & 80 \\
\hline
\end{tabular}

\section{FIGURES}

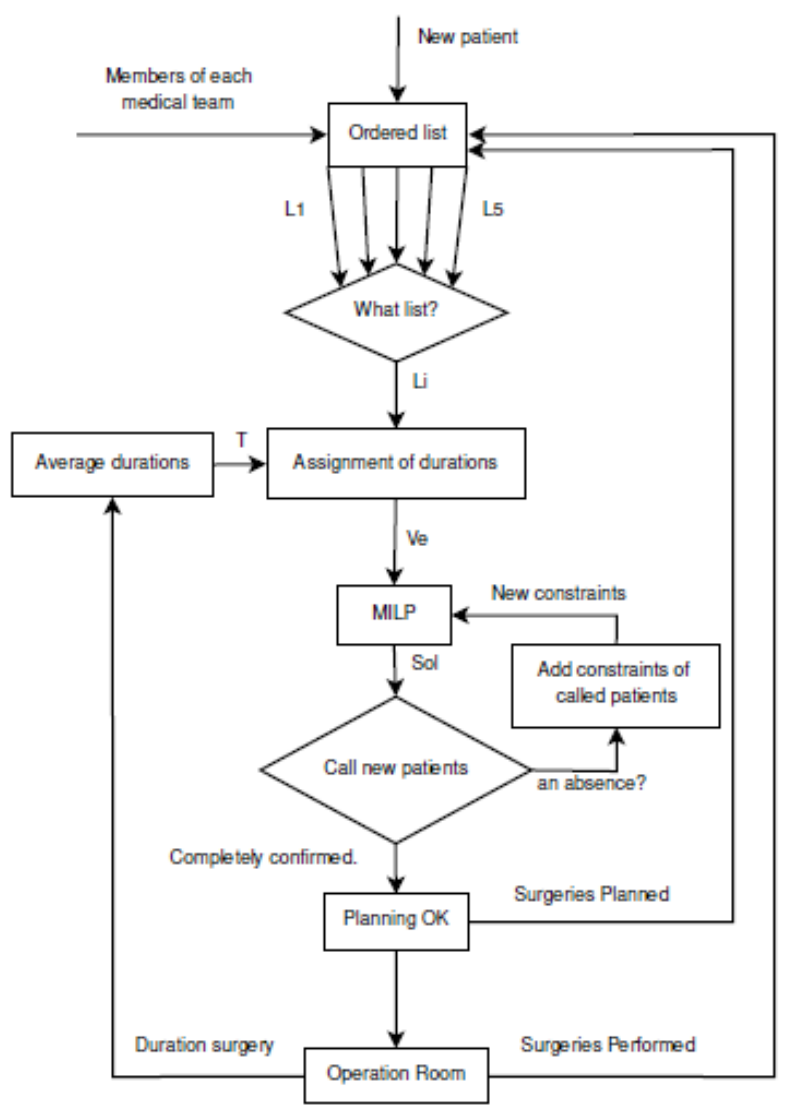

Figura 1. Folwchart of the DSS for operation planning. 